\title{
ON BARRELLED TOPOLOGICAL MODULES
}

\section{DINAMÉRICO P. POMBO JR.*}

Instituto de Matemática

Universidade Federal do Rio de Janeiro

Caixa Postal 68530

21945 Rio de Janeiro Brasil

(Received September 14, 1992 and in revised form February 5, 1993)

ABSTRACT. Some of the main theorems concerning ultrabarrelled topological vector spaces are extended to the context of topological modules.

KEYWORDS AND PHRASES. Topological modules, Banach-Steinhaus theorem, Open Mapping and Closed Graph theorems.

1991 MATHEMATICS SUBJECT CLASSIFICATION CODE. $46 \mathrm{H} 25$

\section{INTRODUCTION AND PRELIMINARIES}

The study of (real or complex) ultrabarrelled topological vector spaces, the topological vector spaces which replace barrelled locally convex spaces when local convexity is not presupposed, was initiated by W. Robertson in [1]. Since then, various authors have been considering the subject. The most important results concerning ultrabarrelled topological vector spaces may be found in the texts [2] and [3], the latter dealing with the case in which the fields of real or complex numbers are replaced by a non-trivially valued division ring.

In this article we introduce and study the concept of barrelled topological module, the natural extension of the classical concept of ultrabarrelled topological vector space. The main results obtained here are extensions of the Banach-Steinhaus theorem and of the Open Mapping and Closed Graph theorems to the context of topological modules. A version of Bourbaki's criterion for the equicontinuity of separately equicontinuous families of bilinear mappings and a version of Grothendieck's "Théorème B" are also established. It should also be mentioned that the methods used by W. Robertson in her fundamental article just cited have strongly influenced the preparation of our article.

We shall adopt the terminology of [4]. Throughout, $A$ denotes an arbitrary topological ring, unless otherwise specified. If $A$ has an identity element, $A^{\star}$ denotes the multiplicative group of its invertible elements. For every $A$-modules $E$ and $F, \mathcal{F}(E ; F)$ denotes the $A$-module of 
all mappings from $E$ into $F$ and $\mathcal{L}_{a}(E ; F)$ denotes the additive sulggroup of $\mathcal{F}(E ; F)$ of all $A$ linear mappings from $E$ into $F$. For every topological $A$-modules $E$ and $F, \mathcal{L}(E ; F)$ denotes the subgroup of $\mathcal{L}_{a}(E ; F)$ of all continuous $A$-linear mappings from $E$ into $F$. For cvery topological $A$-modules $E, F$ and $G, \mathcal{L}_{\text {sep }}(E, F ; G)$ denotes the additive group of all separately continuous $A$ bilinear mappings from $E \times F$ into $G$. If $f \in \mathcal{L}_{\text {sep }}(E, F ; G), x \in E, y \in F, f_{x}$ (1csp. $\left.f_{y}\right)$ denotes the continuous $A$-linear mapping $v \in F \mapsto f_{x}(v)=f(x, v) \in G$ (resp). $u \in E \mapsto f_{y}(u)=f(u, y) \in G$ ). If $\mathcal{X} \subset \mathcal{L}_{s e p}(E, F ; G), x \in E, y \in F, x_{x}:=\left\{f_{x} ; f \in \mathfrak{l}^{\prime}\right\}$ and $x_{y}:=\left\{f_{y} ; f \in \mathfrak{x}^{\prime}\right\}$.

\section{THE CLASS OF BARRELLED TOPOLOGICAL $A$-MODULES}

Definition 2.1. A topological $A$-module $(E, \tau)$ is said to be barrelled if every $A$-module topology on $E$ which admits a fundiumental system of neighlorhoods of the origin consisting of $\tau$-closed sets is weaker than $\tau$.

Remark 2.1. Assume that $A$ is endowed with the discrete topology and let $(E, \tau)$ be a separated topological $A$-module. Then $E$ is barrelled if and only if $\tau$ is discrete.

Proposition 2.1. Let $A$ be a separated topological ring and let $E$ be a separated barrelled - topological $A$-module. Then the completion $\widehat{E}$ of $E$ is a barrelled topological $\widehat{A}$-module, where $\hat{A}$ designates the completion of $A$.

Proof. Analogous to that of Propositon 14 of [1].

The following information will be needed in the sequel.

Proposition 2.2. Let $\left(E_{\imath}\right)_{i \in I}$ be a family of topological $A$-modules, $E$ an $A$-module and, for each $i \in I$, let $f_{2} \in \mathcal{L}_{a}\left(E_{i} ; E\right)$. Then there exists a unique $A$-module topology on $E$ which is final for the family $\left(E_{\imath}, f_{\imath}\right)_{\imath \in I}$. In particular, inductive limits exist in the category of topological $A$-modules.

Proof. Analogous to that of Theorem 2 of [5].

We now present certain stability properties of the class of barrelled topological $A$-modules, some of which have been obtained in [1] (Proposition 13) and [6] (Corollary 1, p.297) when $A=\mathbf{R}$ or $\mathbf{C}$.

Proposition 2.3. Let $\left(\left(E_{i}, \tau_{i}\right)\right)_{i \in I}$ be a family of barrelled topological $A$-modules. Let $E$ be an $A$-module and, for each $i \in I$, let $f_{i} \in \mathcal{L}_{a}\left(E_{i} ; E\right)$. Let $\tau$ be the final $A$-module topology for the family $\left(\left(E_{i}, \tau_{\imath}\right), f_{z}\right)_{i \in I}$ (Proposition 2.2). Then $(E, \tau)$ is barrelled.

Proof. Let $\tau^{*}$ be an $A$-module topology on $E$ which admits a fundamental system $\mathcal{V}$ of neighborhoods of 0 consisting of $\tau$-closed sets. Fix an $i \in I$ and let $\mathcal{B}_{i}$ be the filter base on $E_{i}$ formed by the sets $f_{i}^{-1}(V)(V \in \mathcal{V})$. By Theorem 12.3 of [4] there is a unique $A$-module topology $\tau_{i}^{*}$ on $E_{z}$ for which $\mathcal{B}_{i}$ is a fundamental system of neighborhoods of 0 . Since each $f_{z}^{-1}(V)$ is $\tau_{z}$-closed and since $\left(E_{i}, \tau_{i}\right)$ is barrelled, it follows that $\tau_{i}^{*} \subset \tau_{i}$. Hence $f_{i}:\left(E_{i}, \tau_{i}\right) \rightarrow\left(E, \tau^{*}\right)$ is continuous. By the arbitrariness of $i$ we obtain $\tau^{*} \subset \tau$, and so $(E, \tau)$ is barrelled.

Corollary 2.1. (a) An inductive limit of an inductive system of barrelled topological $A$-modules is a barrelled topological $A$-module.

(b) A quotient by a submodule of a barrelled topological $A$-module is a barrelled topological $A$-module. 
(c) For a direct sum of a family of topological $A$-modules to be barrelled it is necessary and sufficient that each of its members be barrelled.

Proof. (a), (b) and the sufficiency of (c) are immediate consequences of Proposition 2.3. The necessity of (c) follows from (b).

The following proposition contains Theorem 2.37 of [3] (he'nce Proposition 12 of [1]) as a particular case.

Proposition 2.4. Let $A$ be a topological ring with identity and assume that there exists a countable subset $C$ of $A^{\star}$ such that $0 \in \bar{C}$. If $(E, \tau)$ is a unitary topological $A$-module and $M$ is a submodule of $E$ which is non-meager in $(E, \tau)$, then $M$ is barrelled under the induced topology. In particular, every Baire unitary topological $A$-module is barrelled.

Proof. Let $\tau^{\prime}$ be the $A$-module topology on $M$ induced by $\tau$, and let $\tau^{*}$ be an $A$-module topology on $M$ which admits a fundamental system $\mathcal{V}$ of neighborhoods of 0 consisting of $\tau^{\prime}$-closed sets. Given an arbitrary $V \in \mathcal{V}$ there is a $U \in \mathcal{V}$ with $U-U \subset V$. Since $0 \in \bar{C}$, there is a sequence $\left(a_{n}\right)_{n \in \mathbf{N}}$ in $A^{\star}$ such that

$$
M \subset \bigcup_{n \in \mathbf{N}} a_{n} \overline{U^{\tau}}
$$

Therefore some $a_{m} \overline{U^{\tau}}$ has a non-empty interior, because each $a_{n} \overline{U^{\tau}}$ is $\tau$-closed and $M$ is nonmeager in $(E, \tau)$. Hence $\overline{U^{\tau}}$ has a non-empty interior, since the mapping $x \in(E, \tau) \mapsto a_{m} x \in$ $(E, \tau)$ is a homeomorphism. If $x \in \operatorname{int}\left(\overline{U^{\tau}}\right)$, there is a neighborhood $U^{\prime}$ of 0 in $(E, \tau)$ with $x+U^{\prime} \subset \overline{U^{\tau}}$. Consequently, $U^{\prime} \subset \overline{U^{\tau}}-\overline{U^{\tau}} \subset \overline{V^{\tau}}$, and so $\overline{V^{\tau}}$ is a neighborhood of 0 in $(E, \tau)$. Thus $V$ is a neighborhood of 0 in $\left(M, \tau^{\prime}\right)$, because $V=\overline{V^{\tau^{\prime}}}=M \cap \overline{V^{\tau}}$. Therefore $\tau^{*} \subset \tau^{\prime}$, and so $\left(M, \tau^{\prime}\right)$ is barrelled.

Remark 2.2. (a) If $A$ has an identity element, then the relation $0 \in \overline{A^{\star}}$ implies that the topology of $A$ is non-discrete (and is equivalent to this fact when $A$ is a topological division ring).

(b) Every topological division ring which possesses a null sequence of non-zero elements satisfies the hypotheses of Proposition 2.4. Every topological ring with identity which contains an invertible topologically nilpotent element satisfies the hypotheses of Proposition 2.4 .

\section{THE BANACH-STEINHAUS THEOREM AND SOME CONSEQUENCES}

The Banach-Steinhaus theorem holds in our setting (previous results in this direction may be found in [1], Theorem 5; [7], Theorem 3; [3], Theorem 2.58; [4], Theorem 25.6):

Theorem 3.1. Let $E$ be a barrelled topological $A$-module, $F$ an arbitrary topological $A$-module and $\mathcal{X} \subset \mathcal{L}(E ; F)$. If $\mathcal{X}(x)=\{f(x) ; f \in \mathcal{X}\}$ is bounded in $F$ for each $x \in E$, then $\mathcal{X}$ is equicontinuous.

Proof. Let $\tau$ be the given topology of $E$ and let $\mathcal{V}$ be a funclamental system of closed neighborhoods of 0 in $F$. For each $V \in \mathcal{V}$, let

$$
U_{V}=\bigcap_{f \in \mathcal{X}} f^{-1}(V)
$$


Obviously, $\mathcal{B}=\left(U_{V}\right)_{V \in V}$ is a filter basse on $E$. By Theorem 12.3 of [4] there is a unique $A$-module topology $\tau^{*}$ on $E$ for which $\mathcal{B}$ is a fundamental system of neighborhoods of 0 (the condition (TMN 2) of Theorem 12.3 holds because the sets $\mathcal{x}^{\prime}(x)$ are bounded in $\left.F\right)$. Since $(E, \tau)$ is barrelled and since each $U_{V}$ is $\tau$-closed, it follows that $\tau^{*} \subset \tau$. Thus each $U_{V}$ is a neighborhood of 0 in $(E, \tau)$, and so $\mathcal{X}$ is equicontinuous.

Corollary 3.1. Under the assumptions of Theorem 3.1, assume additionally that $F$ is separated. Let $\left(f_{\mathfrak{z}}\right)_{\mathrm{z} \in I}$ be a net in $\mathcal{L}(E ; F)$ such that $\left(f_{\mathfrak{z}}(x)\right)_{\mathrm{a} \in I}$ is bounded in $F$ for each $x \in E$ and such that $\left(f_{i}\right)_{\imath \in I}$ is pointwise convergent to a mappings $f: E \rightarrow F$. Then $f \in \mathcal{L}(E ; F)$ and $\left(f_{i}\right)_{\mathfrak{z} \in I}$ converges to $f$ uniformly on every precompact sulset of $E$. In particular, if $\left(f_{n}\right)_{n \in \mathbb{N}}$ is a sequence in $\mathcal{L}(E ; F)$ pointwise convergent to a mapping $f: E \rightarrow F$, then $f \in \mathcal{L}(E ; F)$ and $\left(f_{n}\right)_{n \in \mathbf{N}}$ converges to $f$ uniformly on every precompact subset of $E$.

Proof. By Theorem 3.1, $\left(f_{\imath}\right)_{\imath \in I}$ is an equicontinuous net. Thus it suffices to apply Proposition 6 and Theorem 1 of [8], chap.X, $§ 2$, and the fact that $\mathcal{L}_{a}(E ; F)$ is simply closed in $\mathcal{F}(E ; F)$, to conclude the proof.

Remark 3.1. Let $E$ and $F$ be topological $A$-modules and let $\mathcal{B}$ be a family of bounded subsets of $E$. Then the topology of $\mathcal{B}$-convergence on $\mathcal{L}(E ; F)$ (denoted by $\tau_{\mathcal{B}}$ ) is an additive group topology which is separated if $\mathcal{B}$ is a covering of $E$ and $F$ is separated. Moreover, if $A$ is commutative, then $\mathcal{L}(E ; F)$ is an $A$-module and $\tau_{\mathcal{B}}$ is an $A$-module topology on $\mathcal{L}(E ; F)$ ([9], Proposition (a)). When $\mathcal{B}$ is the family of all finite (resp. bounded) sulssets of $E$, we write $\tau_{\mathcal{B}}=\tau_{s}$ (resp. $\tau_{\mathcal{B}}=\tau_{b}$ ).

Corollary 3.2. Let $A$ be a commutative topological ring with identity such that $0 \in \overline{A^{\star}}$. Let $E$ be a barrelled topological $A$-module, and let $F$ be a separated locally compact unitary topological $A$-module. If $\mathcal{X} \subset \mathcal{L}(E ; F)$, the following statements are equivalent:

(i) $\mathcal{X}$ is $\tau_{b}$-bounded in $\mathcal{L}(E ; F)$;

(ii) $\mathcal{X}$ is $\tau_{s}$-bounded in $\mathcal{L}(E ; F)$;

(iii) $\mathcal{X}$ is $\tau_{s}$-relatively compact in $\mathcal{L}(E ; F)$;

(iv) $\mathcal{X}$ is equicontinuous.

In order to prove Corollary 3.2 we shall need a lemma which is an extension of the AlaogluBourbaki theorem:

Lemma 3.1. Let $A$ be a topological ring with identity such that $0 \in \overline{A^{\star}}$. Let $E$ be a topological $A$-module and let $F$ be a separated locally compact unitary topological $A$-module. If $\mathcal{X} \subset \mathcal{L}(E ; F)$ is equicontinuous, then $\mathcal{X}$ is $\tau_{s}$-relatively compact in $\mathcal{L}(E ; F)$.

Proof. Since $\mathcal{L}_{a}(E ; F)$ is simply closed in $\mathcal{F}(E ; F)$ and since $\mathcal{X}$ is equicontinuous, it suffices to verify that $\mathcal{X}$ is simply relatively compact in $\mathcal{F}(E ; F)$. But, for each $x \in E, \mathcal{X}(x)$ is bounded in $F$ ([4], Theorem 25.5), hence relatively compact in $F$ ([4], Theorem $15.4(3))$. By Tychonoff's theorem, $\mathcal{X}$ is simply relatively compact in $\mathcal{F}(E ; F)$.

Proof of Corollary 3.2. (i) $\Rightarrow$ (ii): Obvious; (ii) $\Rightarrow$ (iii): Theorem 3.1 and Lemma 3.1; (ii) $\Rightarrow$ (iv): Theorem 15.4 (1) of [4] and Theorem 3.1; (iv) $\Rightarrow$ (i): Theorem 25.5 of [4].

Non-locally convex versions of a classical theorem of Bourbaki ([10], TVS III. 29) have been obtained in [7] (Theorem 5) and [11] (Corollary 9). Our next goal is to prove that it remains valid in our context. 
Theorem 3.2. Let $E$ and $F$ be metrizable topological $A$-modules, $E$ being assumed barrelled, and let $G$ be an arbitrary topological $A$-module. If $x^{\prime} \subset \mathcal{L}_{s c p}(E, F ; G)$ is such that $x_{x}$ is equicontinuous for each $x \in E$, then $\mathcal{X}$ is equicontinuous.

In order to prove the above theorem we shall need two lemmas.

Lemma 3.2. Let $E, F$ and $G$ be topological $A$-modules, and let $\mathcal{X}$ be a separately equicontinuous family of $A$-bilinear mappings from $E \times F$ into $G$. If $\mathcal{x}$ is equicontinuous at the origin, then $x^{\prime}$ is equicontinuous.

Proof. It suffices to recall the identity

$$
f(x, y)-f\left(x_{o}, y_{o}\right)=f\left(x-x_{o}, y-y_{o}\right)+f\left(x-x_{o}, y_{o}\right)+f\left(x_{o}, y-y_{o}\right)
$$

which holds for every $A$-bilinear mapping $f: E \times F \rightarrow G$ and every points $(x, y),\left(x_{o}, y_{o}\right)$ in $E \times F$.

Remark 3.2. When $A$ is a non-trivially valued field, the equicontinuity of a family of $A$-bilinear mappings at the origin is sufficient to ensure its equicontinuity ([10], Proposition 6, TVS I.9).

Lemma 3.3. Let $E, F$ and $G$ be topological $A$-modules, $E$ being assumed barrelled. Let $\mathcal{X} \subset \mathcal{L}_{\text {sep }}(E, F ; G)$ be such that $\mathcal{X}_{x}$ is equicontinous for each $x \in E$. Then $\mathcal{X}_{y}$ is equicontinuous for each $y \in F$.

Proof. Fix a $y \in F$. If $x \in E, \mathfrak{x}_{y}(x)=\mathcal{X}_{x}(y)$ is bounded in $G$ since $\mathcal{X}_{x}$ is equicontinuous. By Theorem $3.1 \mathcal{X}_{y}$ is equicontinuous.

Proof of Theorem 3.2. In view of Lemmas 3.2 and 3.3, it is enough to establish the equicontinuity of $\mathcal{X}$ at $(0,0)$. If $\mathcal{X}$ is not equicontinuous at $(0,0)$, there are a neighborhood $W$ of 0 in $G$, a null sequence $\left(x_{n}\right)_{n \in \mathbb{N}}$ in $E$, a null sequence $\left(y_{n}\right)_{n \in \mathbb{N}}$ in $F$ and a sequence $\left(f_{n}\right)_{n \in \mathbb{N}}$ in $\mathcal{X}$ such that $f_{n}\left(x_{n}, y_{n}\right) \notin W$ for all $n \in \mathbf{N}$ (remember that $E$ and $F$ are metrizable). By Theorem 25.5 of [4] and Theorem 3.1, the family

$$
\left\{f_{y_{n}} ; f \in \mathcal{X}, n \in \mathbf{N}\right\}
$$

is equicontinous. Therefore, there is an integer $n_{o}$ such that $f\left(x_{n}, y_{n}\right) \in W$ for all $f \in \mathcal{X}$ and all $n \geq n_{o}$, a contradiction. Thus $\mathcal{X}$ is equicontinuous.

From Theorems 3.1 and 3.2 we derive:

Corollary 3.3. Let $E$ and $F$ be barrelled metrizable topological $A$-modules, and let $G$ be an arbitrary topological $A$-module. If $\mathcal{X} \subset \mathcal{L}_{\text {sep }}(E, F ; G)$ is such that $\mathcal{X}(x, y)$ is bounded in $G$ for all $(x, y) \in E \times F$, then $\mathcal{X}$ is equicontinuous. 


\section{OPEN MAPPING AND CLOSED GRAPH THEOREMS}

The following theorem is an extension of Theorems 2.49 and 2.73 of [3], hence of the Open Mapping and Closed Graph theorems of W. Robertson ([1], Proposition 5).

Theorem 4.1. Let $E$ be a separated barrelled topological $A$-module, and let $F$ be a complete metrizable topological $A$-module.

(a) If $f: F \rightarrow E$ is a continuous surjective $A$-linear mapping, then $f$ is open.

(b) If $f: E \rightarrow F$ is an $A$-linear mapping with a closed graph, then $f$ is continuous.

Proof. Analogous to that of W. Robertson.

We now obtain the Open Mapping and the Closed Graph theorems of Banach ([12], Chap.III, §3) in our setting (see also [4], Theorem 12.17).

Corollary 4.1. Let $A$ be a topological ring with identity, and assume that there exists a countable subset $C$ of $A^{\star}$ such that $0 \in \bar{C}$. Let $E$ and $F$ be metrizable unitary topological $A$-modules, $E$ being assumed complete, and let $f \in \mathcal{L}_{a}(E ; F)$.

(a) If $f$ is continuous and $f(E)$ is non-meager in $F$, then $f$ is open, $f(E)=F$ and $F$ is complete.

(b) If $F$ is complete and $f$ has a closed graph, then $f$ is continuous.

Proof. (a): Since $f(E)$ is a barrelled topological $A$-module under the induced topology (Proposition 2.4), Theorem 4.1 (a) implies that if $U$ is a neighborhood of 0 in $E$, then $f(U)$ is a neighborhood of 0 in $f(E)$. Thus $\overline{f(U)}$ is a neighborhood of 0 in $F$, because $f(E)$ is dense in $F$ by Exercise 15.3 (a) of [4] $(\overline{f(E)}$ is an open submodule). By a well known argument ([10], TVS I.19), $f$ is an open mapping. As a consequence, $f$ is surjective ([4], Exercise 15.3 (a)). Finally, the completeness of $F$ is clear.

(b): Immediate from Proposition 2.4 and Theorem 4.1 (b) ((b) also follows from (a), via a straightforward argument).

We now state a version of Grothendieck's "Théorème B" ([13], p.17), whose part (a) contains Exercise 12.7 of [4] as a particular case.

Theorem 4.2. Let $A$ be a topological ring with identity, and assume that there exists a countable subset $C$ of $A^{\star}$ such that $0 \in \bar{C}$. Let $E$ and $F$ be separated unitary topological $A$-modules, and suppose that:

There exists a sequence $\left(E_{n}\right)_{n \in \mathbb{N}}$ of complete metrizable unitary topological $A$-modules and, for each $n \in \mathbf{N}$, there exists an $f_{n} \in \mathcal{L}\left(E_{n} ; E\right)$ such that $E=\bigcup_{n \in \mathbb{N}} f_{n}\left(E_{n}\right)$.

There exists a family $\left(F_{i}\right)_{i \in I}$ of complete metrizable unitary topological $A$-modules and, for each $i \in I$, there exists a $g_{i} \in \mathcal{L}_{a}\left(F_{i} ; F\right)$ such that the topology of $F$ is the final $A$-module topology for the family $\left(F_{\imath}, g_{i}\right)_{i \in I}$.

(a) If $f: E \rightarrow F$ is a surjective continuous $A$-linear mapping, then $f$ is open.

(b) .If $f: F \rightarrow E$ is an $A$-linear mapping with a sequentially closed graph, then $f$ is continuous.

The proof of Theorem 4.2 depends on a lemma which corresponds to Grothendieck's "Théorème $\mathrm{A}$ " ([13], p.16). 
Lemma 4.1. Let $A$ be as in Theorem 4.2, and let $F$ be a scparated unitary topological $A$-module. Let $G$ be a complete motrizalble unitary topological $A$-module, $h \in \mathcal{L}(G ; F),\left(E_{n}\right)_{n \in \mathbf{N}}$ a sequence a complete metrizable unitary topological $A$-modules and, for each $n \in \mathrm{N}$, let $h_{n} \in \mathcal{L}\left(E_{n} ; F\right)$. Suppose that $h(G) \subset \bigcup_{n \in \mathbb{N}} h_{n}\left(E_{n}\right)$. Then there exists an integer $m$ such that $h(G) \subset h_{m}\left(E_{m}\right)$. Moreover, if $h_{m}$ is injective, then there exists a $g \in \mathcal{L}\left(G ; E_{m}\right)$ such that $h=h_{m} \circ g$.

Proof. The proof relies on Corollary 4.1, and is exactly the same as that of Grothendieck.

Proof of Theorem 4.2. We may assume that $\left(E_{n}\right)_{n \in \mathrm{N}}$ is a sequence of sulmodules of $E$ whose union is $E$.

(a): We may assume that $f$ is bijective. Otherwise, let $M$ be the kernel of $f$ (a closed submodule of $E$ ), and consider $E / M$ endowed with the quotient topology. Then $E / M$ is a separated unitary topological $A$-module and the canonical $A$-linear mapping

$$
x+M \in E / M \longmapsto f(x) \in F
$$

is bijective and continuous. Moreover, if $\pi: E \rightarrow E / M$ is the canonical surjective $A$-linear mapping, then $\pi \circ f_{n} \in \mathcal{L}\left(E_{n} ; E / M\right)$ for all $n \in \mathbf{N}$ and $E / M=\bigcup_{n \in \mathbf{N}}\left(\pi \circ f_{n}\right)\left(E_{n}\right)$. Therefore, it is enough to prove that $f^{-1}$ is continuous. In order to do so, let $h_{n}$ be the restriction of $f$ to $E_{n}$ $(n \in \mathrm{N})$ and fix an $i \in I$. Then

$$
\bigcup_{n \in \mathbb{N}} h_{n}\left(E_{n}\right)=F \supset g_{\imath}\left(F_{\imath}\right)
$$

By Lemma 4.1, there are an $m \in \mathbf{N}$ and a $g \in \mathcal{L}\left(F_{z} ; E_{m}\right)$ such that $g_{\imath}=h_{m} \circ g\left(h_{m}\right.$ is injective). Consequently, $f^{-1} \circ g_{\mathfrak{l}}$ is continuous. By the arbitrariness of $i, f^{-1}$ is continuous.

(b): Since the graph of each $f \circ g_{z}$ is sequentially closed, and since $f$ is continuous if and only if $f \circ g_{\mathrm{z}}$ is continuous for all $i \in I$, we may assume that $F$ is complete and metrizable.

Let $H$ be the graph of $f$ endowed with the separated $A$-module topology induced by that of $F \times E$. For each $n \in \mathrm{N}$, let $H_{n}=H \cap\left(F \times E_{n}\right)$. Obviously, each $H_{n}$ is a submodule of $H$ and $H=\bigcup_{n \in \mathrm{N}} H_{n}$. It is easily seen that $H_{n}$ is closed in $F \times E_{n}$ when $F \times E_{n}$ is endowed with the product topology, so that $H_{n}$ is a complete metrizable unitary topological $A$-module under the induced topology $\tau_{n}$. Moreover, the inclusion of $\left(H_{n}, \tau_{n}\right)$ into $H$ is continuous. Hence we can apply (a) to the restriction to $H$ of the projection of $F \times E$ onto $F$ to get the continuity of the mapping $x \in F \mapsto(x, f(x)) \in H$. Therefore $f$ is continuous, by composition.

Remark 4.1. A topological $A$-module $F$ as in Theorem 4.2 is necessarily barrelled (Propositions 2.3 and 2.4).

\section{REFERENCES}

[1] ROBERTSON, W. Completions of Topological Vector Spaces, Proc. London Math. Soc. 8 (1958), 242-257.

[2] ADASCH, N., ERNST, B., KEIM, D. Topological Vector Spaces, Lecture Notes in Mathematics 639, Springer-Verlag, 1978. 
[3] PROLLA, J.B. Topies in Functional Analysis over Valued Division Rings, North-Holland Math. Studies 77, North-Holland Publishing Company, 1982.

[4] WARNER, S. Topological Ficlds, North-Holland Math. Studies 157, North-Holland Publishing Company, 1989.

[5] POMBO Jr., D.P. Linear Topologies and Linear Bornologies on Modules, J. Indian Math. Soc., to appear.

[6] IYAHEN,S.O. On Certain Classes of Linear Topological Spaces, Proc. London Math. Soc. 18 (1968), 285-307.

[7] TOMÁS̆EK, S. M-Barrelled Spaces, Comment. Math.Univ. Carolinae 11 (1970), 185-207.

[8] BOURBAKI, N. General Topology, Part 2, Springer-Verlag, 1987.

[9] POMBO Jr., D.P. On the Completion of Certain Topological Modules, Math. Japonica 37 (1992), 333-336.

[10] BOURBAKI, N. Topological Vector Spaces, Springer-Verlag, 1987.

[11] POMBO Jr., D.P. Polynomials in Topological Vector Spaces over Valued Fields, Rend. Circ. Mat. Palermo 37 (1988), 416-430.

[12] BANACH, S. Théorie des Opérations Linéaires, Warszawa, 1932.

[13] GROTHENDIECK, A. Produits Tensoriels Topologiques et Espaces Nucléaires, Mem. Amer. Math. Soc. 16, 1955. 


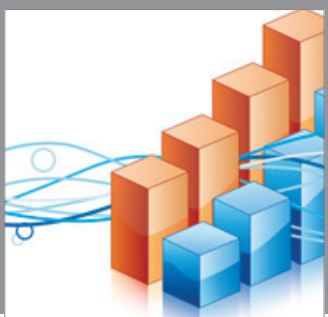

Advances in

Operations Research

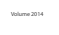

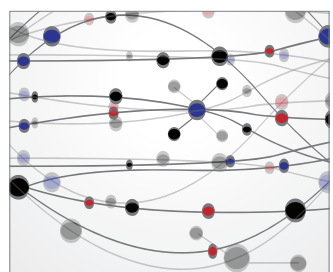

\section{The Scientific} World Journal
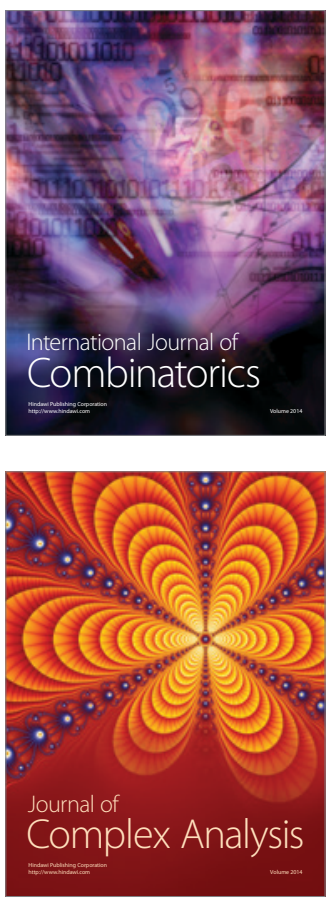

International Journal of

Mathematics and

Mathematical

Sciences
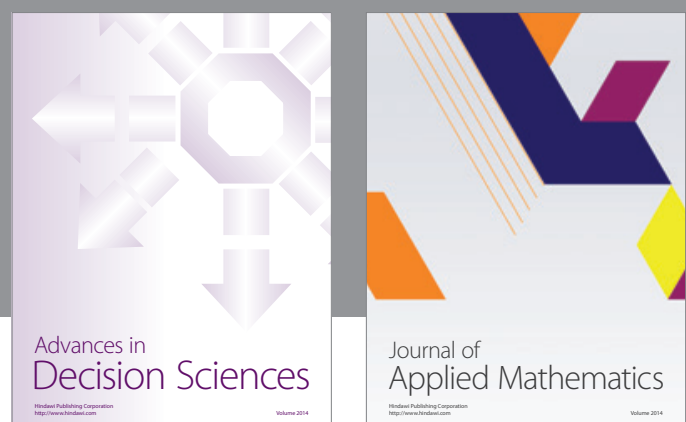

Journal of

Applied Mathematics
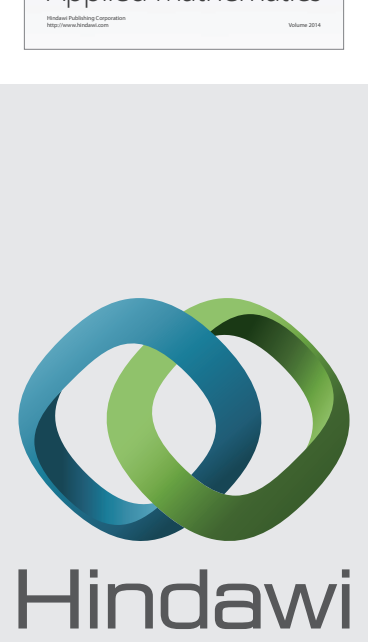

Submit your manuscripts at http://www.hindawi.com
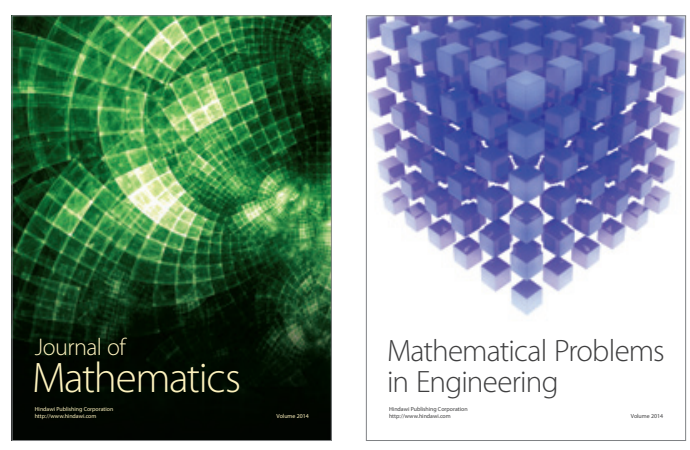

Mathematical Problems in Engineering
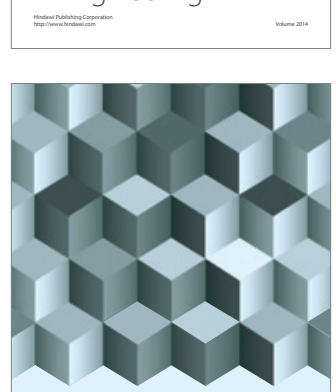

Journal of

Function Spaces
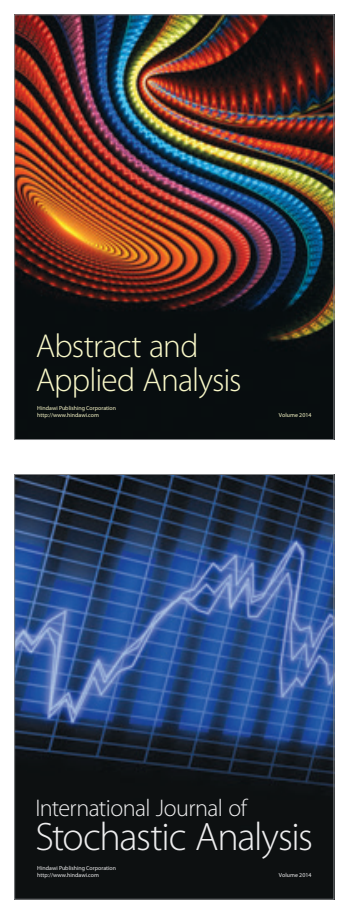

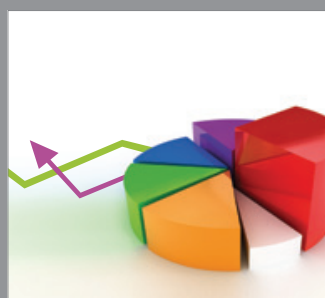

ournal of

Probability and Statistics

Promensencen
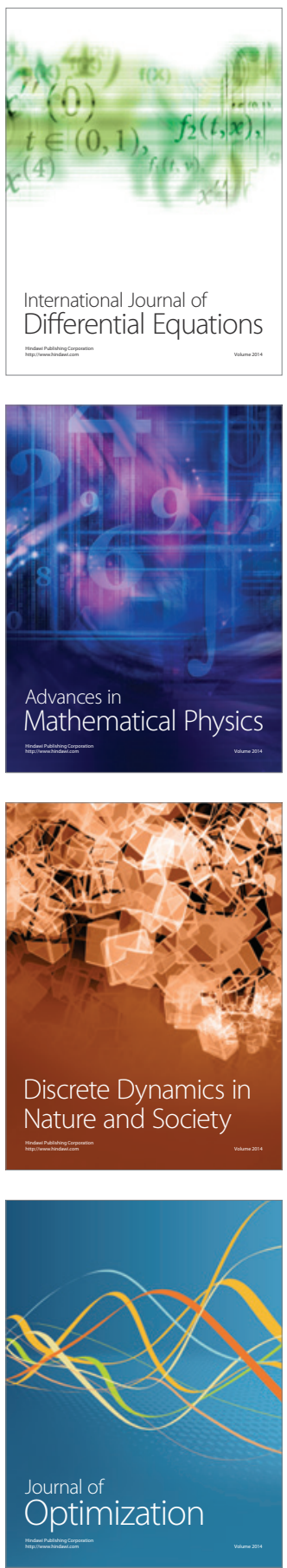